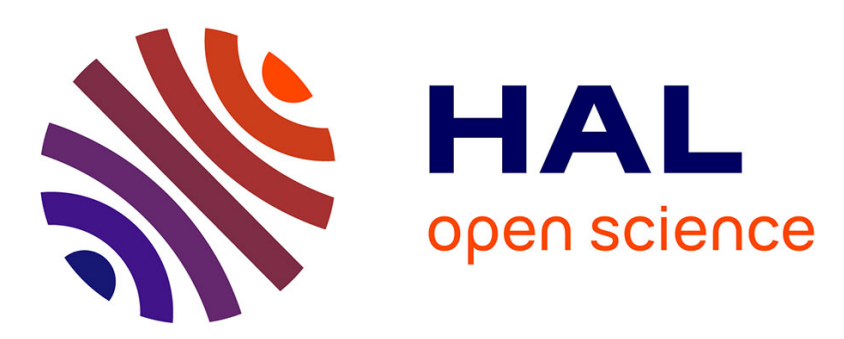

\title{
Transient response of thermoelastic bodies linked by a thin layer of low stiffness and high thermal resistivity
}

\author{
Christian Licht, Ahmed Ould Khaoua, Thibaut Weller
}

\section{To cite this version:}

Christian Licht, Ahmed Ould Khaoua, Thibaut Weller. Transient response of thermoelastic bodies linked by a thin layer of low stiffness and high thermal resistivity. Comptes Rendus Mécanique, 2015, 343 (1), pp.18-26. 10.1016/j.crme.2014.09.005 . hal-01232839

\section{HAL Id: hal-01232839 \\ https://hal.science/hal-01232839}

Submitted on 30 Nov 2015

HAL is a multi-disciplinary open access archive for the deposit and dissemination of scientific research documents, whether they are published or not. The documents may come from teaching and research institutions in France or abroad, or from public or private research centers.
L'archive ouverte pluridisciplinaire HAL, est destinée au dépôt et à la diffusion de documents scientifiques de niveau recherche, publiés ou non, émanant des établissements d'enseignement et de recherche français ou étrangers, des laboratoires publics ou privés. 


\title{
Transient response of thermoelastic bodies linked by a thin layer of low stiffness and high thermal resistivity
}

\section{Réponse instationnaire de corps thermoélastiques liées par une couche mince de faible rigidité et haute résistivité thermique}

\author{
Christian Licht ${ }^{\mathrm{a}, \mathrm{b}, \mathrm{c}}$, Ahmed Ould Khaoua ${ }^{\mathrm{d}}$, Thibaut Weller ${ }^{\mathrm{a}, *}$
}

a LMGC, UMR-CNRS 5508, Université Montpellier-2, case courier 048, place Eugène-Bataillon, 34095 Montpellier cedex 5, France

b Department of Mathematics, Faculty of Science, Mahidol University, Bangkok 10400, Thailand

c Centre of Excellence in Mathematics, CHE, Bangkok 10400, Thailand

d Departamento de Matemáticas, Universidad de los Andes, Cra 1 No 18A-12, Bogota, Colombia

\section{A B S T R A C T}

We extend to the thermoelastic case the study [1] devoted to the dynamic response of a structure made of two linearly elastic bodies linked by a thin soft adhesive linearly elastic layer. Once again, a formulation in terms of an evolution equation in a Hilbert space of possible states with finite energy makes it possible to identify the asymptotic behavior, when some geometrical and thermomechanical parameters tend to their natural limits, as the response of two bodies linked by a thermomechanical constraint. The genuine thermomechanical coupling remains in the constraint law only for a specific relative behavior of the parameters.

\section{R É S U M É}

On étend au cas thermoélastique l'étude [1] consacrée à la réponse dynamique d'un assemblage de deux corps linéairement élastiques liés par une couche adhésive linéairement élastique mince et molle. À nouveau, une formulation en terme d'équations d'évolution dans un espace de Hilbert d'états possibles d'énergie finie permet d'identifier le comportement asymptotique, lorsque des paramètres géométriques et thermomécaniques tendent vers leurs limites naturelles, comme la réponse de l'assemblage des deux corps par une liaison thermomécanique. Le couplage thermomécanique initial perdure dans la loi de la liaison uniquement pour un comportement relatif particulier des paramètres.

\footnotetext{
* Corresponding author.

E-mail addresses: clicht@univ-montp2.fr (C. Licht), aould@uniandes.edu.co (A. Ould Khaoua), thibaut.weller@univ-montp2.fr (T. Weller).
} 


\section{Introduction}

Adhesively bonded joints are an attractive way to put together the components of a structure. As in several situations thermal effects are not negligible, we extend a previous study [1] devoted to a linearly elastic material to the linearly thermoelastic case. Taking advantage of the coupling between mechanical and thermal effects, it is still possible to formulate the problem of determining the transient response of a structure made of two linearly thermoelastic bodies perfectly connected by a thin soft thermoelastic layer with high thermal resistivity in terms of an evolution equation in a Hilbert space of possible states (displacement, temperature, velocity) with finite energy. Hence it is possible to adopt the strategy of [1,2] in order to, first, obtain existence and uniqueness results and, then, to study the asymptotic behavior when some geometrical and thermomechanical data, now regarded as parameters, tend to their natural limits. The limit behavior which supports our proposal of a simplified but accurate enough model for the initial physical situation, corresponds to the dynamic response to the initial load of two linearly thermoelastic bodies connected by a thermomechanical constraint along the surface the adhesive layer shrinks to. The structure of the constitutive equations of the constraint is similar to the one of the layer with coefficients depending on the relative behaviors of the parameters but the thermomechanical coupling is maintained only for a particular relative behavior.

\section{Setting the problem}

We consider a structure consisting of two thermoelastic bodies (adherents) bonded by a thin thermoelastic layer (adhesive). The entire system occupies the domain $\Omega \subset \mathbb{R}^{3}$ with a Lipschitz-continuous boundary $\partial \Omega$. Let $\left(\Gamma_{0}^{M}, \Gamma_{1}^{M}\right)$ and $\left(\Gamma_{0}^{T}, \Gamma_{1}^{T}\right)$ be two partitions of $\partial \Omega$ with $\mathcal{H}_{2}\left(\Gamma_{0}^{T}\right)>0$ and $\mathcal{H}_{2}\left(\Gamma_{0}^{M}\right)>0$, where $\mathcal{H}_{2}$ is the two dimensional Hausdorff measure. We denote the orthonormal canonical basis of $\mathbb{R}^{3}$, assimilated to the physical Euclidean space, by $\left\{e_{1}, e_{2}, e_{3}\right\}$ and for all $\left(x_{1}, x_{2}, x_{3}\right)$ in $\mathbb{R}^{3}, \hat{x}$ stands for $\left(x_{1}, x_{2}\right)$. The intersection $S$ of $\Omega$ with $\left\{x_{3}=0\right\}$ is supposed to have a positive Hausdorff measure and it is also assumed that there exists $\varepsilon_{0}>0$ such that $B_{\varepsilon_{0}}=\left\{\left(\hat{x}, x_{3}\right) \in \Omega\right.$; $\left.\left|x_{3}\right|<\varepsilon_{0}\right\}$ is equal to $S \times\left(-\varepsilon_{0}, \varepsilon_{0}\right)$. Let $\varepsilon<\varepsilon_{0}$, then the adhesive occupies the layer $B_{\varepsilon}$, while each of the two adherents occupies $\Omega_{\varepsilon}^{ \pm}:=\left\{x \in \Omega ; \pm x_{3}>\varepsilon\right\}$ and let $\Omega_{\varepsilon}=\Omega_{\varepsilon}^{+} \cup \Omega_{\varepsilon}^{-}$. Adherents and adhesive are assumed to be perfectly stuck together along $S_{\varepsilon}=S_{\varepsilon}^{+} \cup S_{\varepsilon}^{-}, S_{\varepsilon}^{ \pm}:=\left\{x \in \Omega ; x_{3}= \pm \varepsilon\right\}$. The structure is clamped on $\Gamma_{0}^{M}$, maintained at a uniform temperature $T_{0}$ on $\Gamma_{0}^{T}$, subjected to body forces of density $f$, to surface forces of density $g_{M}$ on $\Gamma_{1}^{M}$ and to thermal flux $g^{T}$ on $\Gamma_{1}^{T}$.

The whole structure is modeled as linearly thermoelastic in the following way. Let $(\rho, \beta, \alpha, \kappa, a) \in L^{\infty}\left(\Omega ; \mathbb{R} \times \mathbb{R} \times S^{3} \times\right.$ $\left.S^{3} \times \operatorname{Lin}\left(S^{3}\right)\right)$ satisfying

$$
\left\{\begin{array}{l}
\exists\left(\rho_{m}, \beta_{m}, \kappa_{m}, a_{m}\right) \in(0,+\infty)^{4} \\
\rho(x) \geq \rho_{m}, \quad \beta(x) \geq \beta_{m}, \quad \alpha(x) \geq 0, \quad \kappa(x) \xi \cdot \xi \geq \kappa_{m}|\xi|^{2}, \quad \forall \xi \in \mathbb{R}^{3}, \\
a(x) e \cdot e \geq a_{m}|e|^{2}, \quad \forall e \in S^{3}, \quad \text { a.e. } x \in \Omega
\end{array}\right.
$$

where $S^{3}$ is the space of $3 \times 3$ symmetric matrices with the usual inner product and norm denoted by $\cdot$ and $|\cdot|$, as in $\mathbb{R}^{3}$, and $\operatorname{Lin}\left(S^{3}\right)$ is the space of linear mapping from $S^{3}$ to $S^{3}$. The mass density, the specific heat coefficient, the thermal dilatation, the heat conductivity and the elasticity coefficients in the adherents are $\rho, \beta, \alpha, \kappa$ and $a$, respectively, while the positive numbers $\widetilde{\rho}, \widetilde{\beta}, \widetilde{\alpha}, \widetilde{\kappa}, \lambda$ and $\mu$ denote the mass density, the specific heat coefficient, the thermal dilatation, the heat conductivity and the Lamé coefficients in the adhesive assumed to be isotropic and homogeneous. Thus problem $\left(\mathcal{P}_{h}\right)$ of determining the evolution of the assembly involves the quintuplet $h=(\varepsilon, \lambda, \mu, \kappa, \gamma)$ of data where $\gamma=(3 \lambda+2 \mu) \widetilde{\alpha}$ and thereafter all the fields will be indexed by $h$. In the following, the upper dot denotes the differentiation with respect to time $t, e(u)$ is the linearized strain tensor associated with the displacement field $u$. Hence problem $\left(\mathcal{P}_{h}\right)$ reads as:

$$
\left(\mathcal{P}_{h}\right)\left\{\begin{array}{l}
\rho \ddot{u}_{h}=\operatorname{div} \sigma_{h}+f, \quad T_{0} \beta \dot{\theta}_{h}=\operatorname{div} q_{h}-T_{0} a \alpha \cdot e\left(\dot{u}_{h}\right) \quad \text { in } \Omega_{\varepsilon} \\
\sigma_{h}=a\left(e\left(u_{h}\right)-\theta_{h} \alpha\right), \quad q_{h}=\kappa \nabla \theta_{h} \quad \text { in } \Omega_{\varepsilon} \\
\tilde{\rho} \ddot{u}_{h}=\operatorname{div} \sigma_{h}+f, \quad T_{0} \widetilde{\beta} \dot{\theta}_{h}=\operatorname{div} q_{h}-T_{0} \gamma \operatorname{tr} e\left(\dot{u}_{h}\right) \quad \text { in } B_{\varepsilon} \\
\sigma_{h}=\lambda \operatorname{tr} e\left(u_{h}\right) I d+2 \mu e\left(u_{h}\right)-\gamma \theta_{h} I d, \quad q_{h}=\widetilde{\kappa} \nabla \theta_{h} \quad \text { in } B_{\varepsilon} \\
\sigma_{h} v=g^{M} \text { on } \Gamma_{1}^{M}, \quad q_{h} \cdot v=g^{T} \quad \text { on } \Gamma_{1}^{T}, \quad u_{h}=0 \quad \text { on } \Gamma_{0}^{M}, \quad \theta_{h}=0 \quad \text { on } \Gamma_{0}^{T} \\
u_{h}(x, 0)=u_{h}^{0}(x), \quad \dot{u}_{h}(x, 0)=v_{h}^{0}(x), \quad \theta_{h}(x, 0)=\theta_{h}^{0}(x), \quad \text { a.e. } x \in \Omega
\end{array}\right.
$$

where $u_{h}, \theta_{h}, \sigma_{h}$ and $q_{h}$ are the fields of displacement, temperature increment with respect to $T_{0}$, the stress tensor and the heat flux vector, respectively, while $u_{h}^{0}, v_{h}^{0}(x), \theta_{h}^{0}$ are the initial conditions. The symbols Id and $v$ refer to the $3 \times 3$ identity matrix and the outward unitary normal to $\partial \Omega$.

\section{Existence and uniqueness result for $\left(\mathcal{P}_{h}\right)$}

Assuming that

$$
\left(H_{1}\right): \quad\left(f, g^{M}, g^{T}\right) \in C^{0,1}\left([0, T] ; L^{2}\left(\Omega ; \mathbb{R}^{3}\right)\right) \times C^{2,1}\left([0, T] ; L^{2}\left(\Gamma_{1}^{M} ; \mathbb{R}^{3}\right)\right) \times C^{2,1}\left([0, T] ; L^{2}\left(\Gamma_{1}^{T}\right)\right)
$$


we seek $z_{h}:=\left(u_{h}, \theta_{h}\right)$ in the form

$$
z_{h}=z_{h}^{e}+z_{h}^{r}
$$

where $z_{h}^{e}$ is the unique solution to

$$
z_{h}^{e}(t) \in Z_{h} ; \quad \Phi_{h}\left(z_{h}^{e}(t), z\right)=L(t)(z), \quad \forall z \in Z_{h}, \forall t \in[0, T]
$$

with

$$
\left\{\begin{array}{l}
Z_{h}=H_{\Gamma_{0}^{M}}^{1}\left(\Omega ; \mathbb{R}^{3}\right) \times H_{\Gamma_{0}^{T}}^{1}(\Omega) \\
H_{\Gamma_{0}^{M}}^{1}\left(\Omega ; \mathbb{R}^{3}\right):=\left\{v \in H^{1}\left(\Omega ; \mathbb{R}^{3}\right) ; v=0 \text { in the sense of traces on } \Gamma_{0}^{M}\right\} \\
H_{\Gamma_{0}^{T}}^{1}(\Omega):=\left\{v \in H^{1}(\Omega) ; v=0 \text { in the sense of traces on } \Gamma_{0}^{T}\right\}
\end{array}\right.
$$

$$
\left\{\begin{array}{l}
\Phi_{h}\left(z, z^{\prime}\right)=\left(u, u^{\prime}\right)_{h, 1}+\left(\theta, \theta^{\prime}\right)_{h, 3}-\left(u^{\prime}, \theta\right)_{h, 5}+\left(u, \theta^{\prime}\right)_{h, 5}, \quad \forall z=(u, \theta), \forall z^{\prime}=\left(u^{\prime}, \theta^{\prime}\right) \in Z_{h} \\
\left(u, u^{\prime}\right)_{h, 1}:=\int_{\Omega_{\varepsilon}} a e(u) \cdot e\left(u^{\prime}\right) \mathrm{d} x+\int_{B_{\varepsilon}}\left(\lambda \operatorname{tr} e(u) \operatorname{tr} e\left(u^{\prime}\right)+2 \mu e(u) \cdot e\left(u^{\prime}\right)\right) \mathrm{d} x, \quad \forall u, u^{\prime} \in H_{\Gamma_{0}^{M}}^{1}\left(\Omega ; \mathbb{R}^{3}\right) \\
\left(\theta, \theta^{\prime}\right)_{h, 3}:=\int_{\Omega_{\varepsilon}} \frac{\kappa}{T_{0}} \nabla \theta \cdot \nabla \theta^{\prime} \mathrm{d} x+\int_{B_{\varepsilon}} \frac{\tilde{\kappa}}{T_{0}} \nabla \theta \cdot \nabla \theta^{\prime} \mathrm{d} x, \quad \forall \theta, \theta^{\prime} \in H_{\Gamma_{0}^{T}}^{1}(\Omega) \\
(u, \theta)_{h, 5}:=\int_{\Omega_{\varepsilon}} a \alpha \cdot e(u) \theta \mathrm{d} x+\int_{B_{\varepsilon}} \gamma \operatorname{tr} e(u) \theta \mathrm{d} x, \quad \forall(u, \theta) \in H_{\Gamma_{0}^{M}}^{1}\left(\Omega ; \mathbb{R}^{3}\right) \times H_{\Gamma_{0}^{T}}^{1}(\Omega)
\end{array}\right.
$$

and

$$
L(t)(z):=\int_{\Gamma_{1}^{M}} g^{M}(x, t) \cdot u(x) \mathrm{d} \mathcal{H}_{2}+\int_{\Gamma_{1}^{T}} g^{T}(x, t) \theta(x) \mathrm{d} \mathcal{H}_{2}, \quad \forall z=(u, \theta) \in Z_{h}
$$

As $\left(g^{M}, g^{T}\right) \rightarrow z_{h}^{e}$ is linear continuous from $L^{2}\left(\Gamma_{1}^{M} ; \mathbb{R}^{3}\right) \times L^{2}\left(\Gamma_{1}^{T} ; \mathbb{R}\right)$ into $Z_{h}$, we have

$$
z_{h}^{e} \in C^{2,1}\left([0, T] ; Z_{h}\right)
$$

The remaining part $z_{h}^{r}$ will be involved in an evolution equation governed by a $m$-dissipative operator $A_{h}$ in a Hilbert space of possible states "with finite energy" defined by

$$
H_{h}:=H_{\Gamma_{0}^{M}}^{1}\left(\Omega ; \mathbb{R}^{3}\right) \times L^{2}(\Omega) \times L^{2}\left(\Omega ; \mathbb{R}^{3}\right)
$$

and endowed with the inner product and its norm defined respectively by:

$$
\left(U^{1}, U^{2}\right)_{h}:=\left(u^{1}, u^{2}\right)_{h, 1}+K_{h}\left(\left(v_{1}, \theta_{1}\right),\left(v_{2}, \theta_{2}\right)\right), \quad\left|U^{i}\right|_{h}^{2}:=\left(U^{i}, U^{i}\right)_{h}, \quad \forall U^{i}=\left(u^{i}, \theta^{i}, v^{i}\right) \in H_{h}, i=1,2
$$

with

$$
\left\{\begin{array}{l}
K_{h}\left((v, \theta),\left(v^{\prime}, \theta^{\prime}\right)\right)=\left(v, v^{\prime}\right)_{h, 2}+\left(\theta, \theta^{\prime}\right)_{h, 4} \\
\left(v, v^{\prime}\right)_{h, 2}:=\int_{\Omega_{\varepsilon}} \rho v \cdot v^{\prime} \mathrm{d} x+\int_{B_{\varepsilon}} \widetilde{\rho} v \cdot v^{\prime} \mathrm{d} x, \quad \forall v, v^{\prime} \in L^{2}\left(\Omega ; \mathbb{R}^{3}\right) \\
\left(\theta, \theta^{\prime}\right)_{h, 4}:=\int_{\Omega_{\varepsilon}} \beta \theta \theta^{\prime} \mathrm{d} x+\int_{B_{\varepsilon}} \tilde{\beta} \theta \theta^{\prime} \mathrm{d} x, \quad \forall \theta, \theta^{\prime} \in L^{2}(\Omega)
\end{array}\right.
$$

Operator $A_{h}$ is defined by:

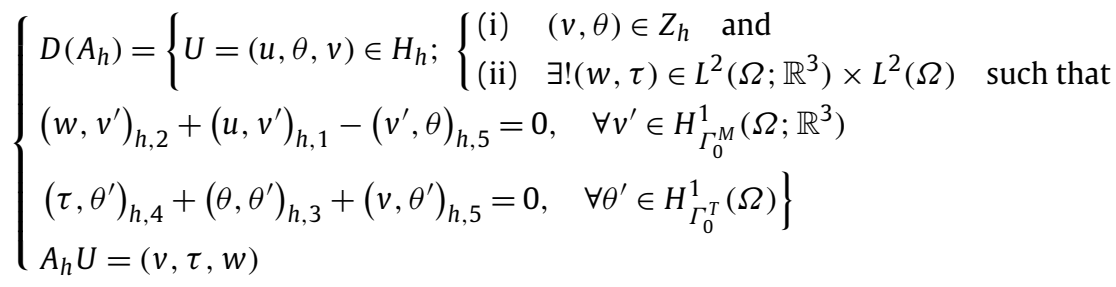

It is straightforward to check the following. 
Proposition 3.1. Operator $A_{h}$ is m-dissipative and for all $\phi_{h}=\left(\phi_{h}^{1}, \phi_{h}^{2}, \phi_{h}^{3}\right)$ in $H_{h}$

$$
\left\{\begin{array} { l } 
{ \overline { U } _ { h } = ( \overline { u } _ { h } , \overline { \theta } _ { h } , \overline { v } _ { h } ) \quad \text { s.t. } } \\
{ \overline { U } _ { h } - A _ { h } \overline { U } _ { h } = \phi _ { h } }
\end{array} \Leftrightarrow \left\{\begin{array}{l}
\bar{u}_{h}=\bar{v}_{h}+\phi_{h}^{1} \\
\bar{z}_{h}=\left(\bar{v}_{h}, \bar{\theta}_{h}\right) \in Z_{h} ; \quad \Psi_{h}\left(\bar{z}_{h}, z\right)=\mathcal{L}_{h}(z), \quad \forall z=(v, \theta) \in Z_{h} \\
\text { with } \\
\Psi_{h}=\Phi_{h}+K_{h} \\
\mathcal{L}_{h}(z)=\left(\phi_{h}^{1}, u\right)_{h, 1}+\left(\phi_{h}^{2}, u\right)_{h, 2}+\left(\phi_{h}^{3}, \theta\right)_{h, 3}, \quad \forall z=(v, \theta) \in Z_{h}
\end{array}\right.\right.
$$

Then taking $\left(H_{1}\right),(3),(4),(8)$ and (12) into account, it is clear that $\left(\mathcal{P}_{h}\right)$ is formally equivalent to

$$
\left\{\begin{array}{l}
\frac{\mathrm{d} U_{h}^{r}}{\mathrm{~d} t}-A_{h} U_{h}^{r}=F_{h}:=\left(u^{e}-\frac{\mathrm{d} u^{e}}{\mathrm{~d} t},-\frac{\mathrm{d} \theta^{e}}{\mathrm{~d} t},-\frac{\mathrm{d} u^{e}}{\mathrm{~d} t}+f_{h}\right) \\
U^{r}(0)=U_{h}^{0}-\left(z_{h}^{e}(0), 0\right), \quad U_{h}^{0}:=\left(u_{h}^{0}, \theta_{h}^{0}, v_{h}^{0}\right)
\end{array}\right.
$$

with

$$
f_{h}=\left(1 / \rho \mathbb{1}_{\Omega_{\varepsilon}}+1 / \widetilde{\rho}\left(1-\mathbb{1}_{\Omega_{\varepsilon}}\right)\right) f
$$

where $\mathbb{1}_{\Omega_{\varepsilon}}$ denotes the characteristic function of $\Omega_{\varepsilon}$. Consequently [3] the following holds:

Theorem 3.1. If $\left(f, g^{M}, g^{T}\right)$ satisfies $\left(H_{1}\right)$ and if $U_{h}^{0}$ belongs to $\left(z_{h}^{e}(0), 0\right)+D\left(A_{h}\right)$, then $(14)$ has a unique solution in $C^{1}\left([0, T]\right.$; $\left.H_{h}\right)$. Hence there exists a unique $\left(u_{h}, \theta_{h}\right)$ in

$$
\left(C^{1}\left([0, T] ; H_{\Gamma_{0}^{M}}^{1}\left(\Omega ; \mathbb{R}^{3}\right)\right) \cap C^{2}\left([0, T] ; L^{2}\left(\Omega ; \mathbb{R}^{3}\right)\right)\right) \times\left(C^{1}\left([0, T] ; L^{2}(\Omega)\right) \cap C^{0}\left([0, T] ; H_{\Gamma_{0}^{T}}^{1}(\Omega)\right)\right)
$$

which satisfies:

$$
\left\{\begin{array}{l}
\left(\frac{\mathrm{d}^{2} u_{h}}{\mathrm{~d} t^{2}}, u\right)_{h, 2}+\left(u_{h}, u\right)_{h, 1}-\left(u, \theta_{h}\right)_{h, 5}=\int_{\Omega} f \cdot u \mathrm{~d} x+\int_{\Gamma_{1}^{M}} g^{M} \cdot u \mathrm{~d} \mathcal{H}_{2}, \quad \forall u \in H_{\Gamma_{0}^{M}}^{1}\left(\Omega ; \mathbb{R}^{3}\right) \\
\left(\frac{\mathrm{d} \theta_{h}}{\mathrm{~d} t}, \theta\right)_{h, 4}+\left(\theta_{h}, \theta\right)_{h, 3}+\left(\frac{\mathrm{d} u_{h}}{\mathrm{~d} t}, \theta\right)_{h, 5}=\int_{\Gamma_{1}^{T}} g^{T} \theta \mathrm{d} \mathcal{H}_{2}, \quad \forall \theta \in H_{\Gamma_{0}^{T}}^{1}(\Omega)
\end{array}\right.
$$

We set:

$$
U_{h}^{e}=\left(z_{h}^{e}, u_{h}^{e}\right)
$$

\section{Asymptotic behavior}

Regarding the quintuplet $h$ of geometrical and thermomechanical data as a quintuplet of parameters taking values in a countable subset of $\left(0, \varepsilon_{0}\right) \times(0,+\infty)^{4}$ with a single cluster point $h^{*}=\left(0, \lambda^{*}, \mu^{*}, \kappa^{*}, \gamma^{*}\right)$, we now study the asymptotic behavior of $z_{h}$ in order to suggest a simplified but accurate enough model for the initial transient thermoelastic problem. We make the following assumptions on the relative magnitudes of the parameters:

$$
\left(H_{2}\right) \quad \begin{cases}\text { i) } & h^{*} \in\{0\} \times[0,+\infty)^{4} \\ \text { ii) } & \exists(\bar{\lambda}, \bar{\mu}, \bar{\kappa}) \in[0,+\infty]^{3} \quad \text { s.t. }(\lambda / 2 \varepsilon, \mu / 2 \varepsilon, \widetilde{\kappa} / 2 \varepsilon) \rightarrow(\bar{\lambda}, \bar{\mu}, \bar{\kappa}) \\ \text { iii) } & \bar{\mu} \in(0,+\infty] \quad \text { if } \min \left\{\mathcal{H}_{2}\left(\Gamma_{0}^{M^{+}}\right), \mathcal{H}_{2}\left(\Gamma_{0}^{M^{-}}\right)\right\}=0 \\ & \bar{\kappa} \in(0,+\infty] \quad \text { if } \min \left\{\mathcal{H}_{2}\left(\Gamma_{0}^{T+}\right), \mathcal{H}_{2}\left(\Gamma_{0}^{T-}\right)\right\}>0 \\ \text { iv) } & \limsup _{s \rightarrow s^{*}} \varepsilon^{2} / \mu=\underset{s \rightarrow s^{*}}{\limsup \varepsilon^{2} / \widetilde{\kappa}=0}\end{cases}
$$

\subsection{A candidate for describing the limit problem}

Depending on the finiteness of $(\bar{\lambda}, \bar{\mu}, \bar{\kappa})$, we will have six distinct cases indexed by $I=\left(I_{1}, I_{2}\right) \in\{1,2,3\} \times\{1,2\}$. The case $I_{1}=1$ corresponds to $(\bar{\lambda}, \bar{\mu}) \in[0,+\infty)^{2}, I_{1}=2$ to $(\bar{\lambda}, \bar{\mu}) \in\{+\infty\} \times[0,+\infty), I_{1}=3$ to $\bar{\mu}=+\infty, I_{2}=1$ to $\bar{\kappa} \in[0,+\infty)$ and $I_{2}=2$ to case $\bar{\kappa}=+\infty$. We introduce the following spaces: 


$$
\begin{aligned}
& { }^{I} Z:={ }^{I_{1}} V \times{ }^{I_{2}} W \\
& { }^{1} V:=H_{\Gamma_{0}^{M}}^{1}\left(\Omega \backslash S ; \mathbb{R}^{3}\right), \quad{ }^{2} V:=\left\{v \in H_{\Gamma_{0}^{M}}^{1}\left(\Omega \backslash S ; \mathbb{R}^{3}\right) ;[v]_{3}=0 \text { on } S\right\}, \quad{ }^{3} V:=H_{\Gamma_{0}^{M}}^{1}\left(\Omega ; \mathbb{R}^{3}\right) \\
& { }^{1} W:=H_{\Gamma_{0}^{T}}^{1}(\Omega \backslash S), \quad{ }^{2} W:=H_{\Gamma_{0}^{T}}^{1}(\Omega)
\end{aligned}
$$

As any element $z=(u, \theta)$ of $H_{\Gamma_{0}^{M}}^{1}\left(\Omega \backslash S ; \mathbb{R}^{3}\right) \times H_{\Gamma_{0}^{T}}^{1}(\Omega \backslash S)$ (spaces defined as $H_{\Gamma_{0}^{M}}^{1}\left(\Omega ; \mathbb{R}^{3}\right)$ and $H_{\Gamma_{0}^{T}}^{1}(\Omega)$ ) has restrictions $z^{ \pm}=\left(u^{ \pm}, \theta^{ \pm}\right)$to $\Omega^{ \pm}$in $H^{1}\left(\Omega^{ \pm} ; \mathbb{R}^{3}\right) \times H^{1}\left(\Omega^{ \pm}\right)$, the difference between the traces on $S$ of $z^{+}$and $z^{-}$, denoted by $[z]=$ $([u],[\theta])$, belongs to $L^{2}\left(S ; \mathbb{R}^{3} \times \mathbb{R}\right)$ and represents the relative displacement and the jump of temperature across $S$.

Let the continuous bilinear forms:

$$
\left\{\begin{array}{l}
{ }^{1}\left(u, u^{\prime}\right)_{1}:=\int_{\Omega \backslash S} a e(u) \cdot e\left(u^{\prime}\right) \mathrm{d} x+\int_{S}\left(\bar{\lambda}[u]_{3}\left[u^{\prime}\right]_{3}+2 \bar{\mu}\left([u] \otimes_{S} e_{3}\right) \cdot\left(\left[u^{\prime}\right] \otimes_{S} e_{3}\right)\right) \mathrm{d} \hat{x}, \quad \forall u, u^{\prime} \in{ }^{1} V \\
{ }^{2}\left(u, u^{\prime}\right)_{1}:=\int_{\Omega \backslash S} a e(u) \cdot e\left(u^{\prime}\right) \mathrm{d} x+\int_{S} 2 \bar{\mu}\left([u] \otimes_{S} e_{3}\right) \cdot\left(\left[u^{\prime}\right] \otimes_{S} e_{3}\right) \mathrm{d} \hat{x}, \quad \forall u, u^{\prime} \in{ }^{1} V \\
{ }^{3}\left(u, u^{\prime}\right)_{1}:=\int_{\Omega} a e(u) \cdot e\left(u^{\prime}\right) \mathrm{d} x, \quad \forall u, u^{\prime} \in{ }^{3} V \\
{ }^{1}\left(\theta, \theta^{\prime}\right)_{3}:=\int_{\Omega \backslash S} \frac{\kappa}{T_{0}} \nabla \theta \cdot \nabla \theta^{\prime} \mathrm{d} x+\int_{S} \frac{\bar{\kappa}}{T_{0}}[\theta]\left[\theta^{\prime}\right] \mathrm{d} \hat{x}, \quad \forall \theta, \theta^{\prime} \in{ }^{1} W \\
{ }^{2}\left(\theta, \theta^{\prime}\right)_{3}:=\int_{\Omega} \frac{\kappa}{T_{0}} \nabla \theta \cdot \nabla \theta^{\prime} \mathrm{d} x, \quad \forall \theta, \theta^{\prime} \in{ }^{2} W \\
\left(u, u^{\prime}\right)_{2}:=\int_{\Omega} \rho u \cdot u^{\prime} \mathrm{d} x, \quad \forall u, u^{\prime} \in L^{2}\left(\Omega ; \mathbb{R}^{3}\right) \\
\left(\theta, \theta^{\prime}\right)_{4}:=\int_{\Omega} \beta \theta \theta^{\prime} \mathrm{d} x, \quad \forall \theta, \theta^{\prime} \in L^{2}(\Omega) \\
{ }^{I}(v, \theta)_{5}:=\int_{\Omega \backslash S} a \alpha \cdot e(v) \theta \mathrm{d} x+\int_{S} \gamma^{*}[v]_{3} \frac{\left(\theta^{+}+\theta^{-}\right)}{2} \mathrm{~d} x, \quad \forall(v, \theta) \in{ }^{I} Z
\end{array}\right.
$$

Hence

$$
{ }^{I} H:=\left\{U=(u, \theta, v) \in{ }^{I} V \times L^{2}(\Omega) \times L^{2}\left(\Omega ; \mathbb{R}^{3}\right)\right\}
$$

is a Hilbert space if equipped with

$$
{ }^{I}|U|^{2}:={ }^{I_{1}}(u, u)_{1}+(v, v)_{2}+(\theta, \theta)_{4}
$$

As for $A_{h}$, it is straightforward to check that operator ${ }^{I} A$ defined by

$$
\left\{\begin{array}{l}
D\left({ }^{I} A\right)=\left\{U=(u, \theta, v) \in{ }^{I} H ; \begin{cases}\text { (i) } & (v, \theta) \in{ }^{I} Z \text { and } \\
\text { (ii) } & \exists !(w, \tau) \in L^{2}\left(\Omega ; \mathbb{R}^{3}\right) \times L^{2}(\Omega) \quad \text { such that } \\
\left(w, v^{\prime}\right)_{2}+{ }^{I_{1}}\left(u, v^{\prime}\right)_{1}-{ }^{I}\left(v^{\prime}, \theta\right)_{5}=0, & \forall v^{\prime} \in{ }^{I_{1}} V \\
\left(\tau, \theta^{\prime}\right)_{4}+{ }^{I_{2}}\left(\theta, \theta^{\prime}\right)_{3}+{ }^{I}\left(v, \theta^{\prime}\right)_{5}=0, & \left.\forall \theta^{\prime} \in{ }^{I_{2}} W\right\} \\
{ }^{I} A U=(v, \tau, w)\end{cases} \right.
\end{array}\right.
$$

is $m$-dissipative and to note that for all $\phi=\left(\phi^{1}, \phi^{2}, \phi^{3}\right)$ in ${ }^{I} H$

$$
\left\{\begin{array} { l } 
{ { } ^ { I } \overline { U } = ( { } ^ { I } \overline { u } , { } ^ { I } \overline { \theta } , { } ^ { I } \overline { v } ) \quad \text { s.t. } } \\
{ { } ^ { I } \overline { U } - { } ^ { I } A ^ { I } \overline { U } = \phi }
\end{array} \Leftrightarrow \left\{\begin{array}{l}
{ }^{I} \bar{u}={ }^{I} \bar{v}+\phi \\
{ }^{I} \bar{z}=\left({ }^{I} \bar{v},{ }^{I} \bar{\theta}\right) \in{ }^{I} Z ; \quad{ }^{I} \Psi\left({ }^{I} \bar{z}, z\right)={ }^{I} \mathcal{L}(z), \quad \forall z \in{ }^{I} Z
\end{array}\right.\right.
$$

with, for any $z=(v, \theta)$ and $z^{\prime}=\left(v^{\prime}, \theta^{\prime}\right)$ in ${ }^{I} Z$ 


$$
\left\{\begin{array}{l}
{ }^{I} \Psi\left(z, z^{\prime}\right):={ }^{I} \Phi\left(z, z^{\prime}\right)+\left(\theta, \theta^{\prime}\right)_{2}+\left(v, v^{\prime}\right)_{4} \\
{ }^{I} \Phi\left(z, z^{\prime}\right):={ }^{I_{1}}\left(v, v^{\prime}\right)_{1}+{ }^{I_{2}}\left(\theta, \theta^{\prime}\right)_{3}-{ }^{I}\left(v^{\prime}, \theta\right)_{5}+{ }^{I}\left(v, \theta^{\prime}\right)_{5} \\
{ }^{I} \mathcal{L}\left(z^{\prime}\right):={ }^{I}\left(\phi^{1}, v^{\prime}\right)_{1}+\left(\phi^{2}, v^{\prime}\right)_{2}+\left(\phi^{3}, \theta^{\prime}\right)_{4}
\end{array}\right.
$$

Thus a similar statement as that of Theorem 3.1 is valid for the following equation, which will be shown to describe the asymptotic behavior of $z_{h}$ :

$$
\left\{\begin{array}{l}
\frac{\mathrm{d}^{I} U^{r}}{\mathrm{~d} t}-{ }^{I} A^{I} U^{r}={ }^{I} F:=\left({ }^{I} u^{e}-\frac{\mathrm{d}^{I} u^{e}}{\mathrm{~d} t},-\frac{\mathrm{d}^{I} \theta^{e}}{\mathrm{~d} t},-\frac{\mathrm{d}^{I} u^{e}}{\mathrm{~d} t}+\frac{f}{\rho}\right) \\
{ }^{I} U^{r}(0)={ }^{I} U^{r, 0}
\end{array}\right.
$$

with

$$
{ }^{I} z^{e}=\left({ }^{I} u^{e},{ }^{I} \theta^{e}\right) \in C^{2,1}\left([0, T],{ }^{I} Z\right) ; \quad{ }^{I} \Psi\left({ }^{I} z^{e}(t), z\right)=L(t)(z), \quad \forall z \in{ }^{I} Z, \forall t \in[0, T]
$$

We set

$$
{ }^{I} U^{e}=\left({ }^{I} z^{e},{ }^{I} u^{e}\right), \quad{ }^{I} U={ }^{I} U^{e}+{ }^{I} U^{r}
$$

\subsection{Convergence}

To prove the convergence of $z_{h}$ toward ${ }^{I} z={ }^{I} z^{e}+{ }^{I} z^{r}$, with ${ }^{I} z^{r}=\left({ }^{I} u^{r},{ }^{I} \theta^{r}\right)$, we use Trotter's theory of convergence of semi-groups of linear operators acting on variable spaces [4] because $z_{h}^{r}$ and ${ }^{I} z^{r}$ do not inhabit the same space.

First, to define a linear operator ${ }^{I} P_{h}$ from ${ }^{I} H$ to $H_{h}$ suitable for comparing the elements of ${ }^{I} H$ and $H_{h}$ we classically $[1,2,5]$ use the smoothing linear continuous operator $R_{\varepsilon}$ from $H^{1}(\Omega \backslash S)$ to $H^{1}(\Omega)$ defined by

$$
R_{\varepsilon} \varphi(x)= \begin{cases}\varphi^{S}(x)+\operatorname{Min}\left\{\left|x_{3}\right| / \varepsilon, 1\right\} \varphi^{A}(x), & \forall x \text { in } B_{\varepsilon} \\ \varphi(x), & \forall x \text { in } \Omega_{\varepsilon}\end{cases}
$$

where $2 \varphi^{S}(x)=\varphi\left(\hat{x}, x_{3}\right)+\varphi\left(\hat{x},-x_{3}\right)$ and $2 \varphi^{A}(x)=\varphi\left(\hat{x}, x_{3}\right)-\varphi\left(\hat{x},-x_{3}\right)$. Hence the operator ${ }^{I} P_{h}$ defined by

$$
\begin{cases}{ }^{I} H \ni U=(u, \theta, v) \mapsto{ }^{I} P_{h} U=\left({ }^{I_{1}} P_{h} u,{ }^{I_{2}} P_{h} \theta, v\right) \in H_{h} \\ I_{1}=1: & \left({ }^{1} P_{h} u\right)_{i}=R_{\varepsilon} u_{i}, \quad i=1,2,3 \\ I_{1}=2: & \left({ }^{2} P_{h} u\right)_{\alpha}=R_{\varepsilon} u_{\alpha}, \quad \alpha=1,2,\left({ }^{2} P_{h} u\right)_{3}=u_{3} \\ I_{1}=3: & { }^{3} P_{h} u=u \\ I_{2}=1: & { }^{1} P_{h} \theta=R_{\varepsilon} \theta \\ I_{2}=2: & { }^{2} P_{h} \theta=\theta\end{cases}
$$

satisfies.

\section{Proposition 4.1.}

i) There exists a strictly positive constant $c$ independent of $h$ such that

$$
\left|{ }^{I} P_{h} U\right|_{h} \leq c^{I}|U|, \quad \forall U \in{ }^{I} H,
$$

ii) when $h$ goes to $h^{*},\left|{ }^{I} P_{h} U\right|_{h}$ converges toward ${ }^{I}|U|$ for all $U$ in ${ }^{I} H$,

iii)

$$
\begin{aligned}
& \left|e\left({ }^{1} P_{h} u\right)-\frac{[u]}{2 \varepsilon} \otimes_{S} e_{3}\right|_{L^{2}\left(B_{\varepsilon} ; S^{2}\right)}^{2} \leq c \varepsilon^{2}|e(u)|_{L^{2}\left(\Omega \backslash S ; S^{3}\right)}^{2}, \quad \forall u \in H_{\Gamma_{0}^{M}}^{1}\left(\Omega \backslash S ; \mathbb{R}^{3}\right) \\
& \left|\nabla\left({ }^{1} P_{h} \theta\right)-\frac{[\theta]}{2 \varepsilon} \otimes_{S} e_{3}\right|_{L^{2}\left(B_{\varepsilon}\right)}^{2} \leq c \varepsilon^{2}|\nabla \theta|_{L^{2}\left(\Omega \backslash S ; \mathbb{R}^{3}\right)}^{2}, \quad \forall \theta \in H_{\Gamma_{0}^{T}}^{1}(\Omega \backslash S) \\
& \left|R_{\varepsilon} \theta-\frac{\gamma_{0}\left(\theta^{+}\right)+\gamma_{0}\left(\theta^{-}\right)}{2}\right|_{L^{2}\left(B_{\varepsilon}\right)}^{2} \leq c \varepsilon^{2}|\nabla \theta|_{L^{2}\left(B_{\varepsilon} ; \mathbb{R}^{3}\right)}^{2}, \quad \forall \theta \in H_{\Gamma_{0}^{T}}^{1}(\Omega \backslash S)
\end{aligned}
$$

where $\gamma_{0}\left(\theta^{ \pm}\right)$denotes the trace on $S$ of $\theta^{ \pm}$. 
Next we state that $U_{h}$ in $H_{h}$ converges in the sense of Trotter toward $U$ in ${ }^{I} H$ if

$$
\lim _{h \rightarrow h^{*}}\left|{ }^{I} P_{h} U-U_{h}\right|=0
$$

As in [5], such a suitable notion of convergence implies the following proposition.

Proposition 4.2. For all $U=(u, \theta, v)$ in ${ }^{I} H$, if $U_{h}=\left(u_{h}, \theta_{h}, v_{h}\right)$ in $H_{h}$ converges toward $U$ in the sense of Trotter, then:

i) $\mathbb{1}_{\Omega_{\varepsilon}}\left(e\left(u_{h}\right), \nabla \theta_{h}\right)$ converges strongly in $L^{2}\left(\Omega \backslash S ; S^{3} \times \mathbb{R}^{3}\right)$ toward $(e(u), \nabla \theta)$ and, for all positive $\eta<\varepsilon_{0}$, the sequence $\left(u_{h}, \theta_{h}\right)$ converges strongly in $H^{1}\left(\Omega_{\eta} ; \mathbb{R}^{3} \times \mathbb{R}\right)$ toward $(u, \theta)$,

ii) the traces on $S_{\varepsilon}^{ \pm}$of $\left(u_{h}, \theta_{h}\right)$ identified with elements of $L^{2}\left(S ; \mathbb{R}^{3} \times \mathbb{R}\right)$, converge strongly in $L^{2}\left(S ; \mathbb{R}^{3} \times \mathbb{R}\right)$ toward the traces on $S$ of $\left(u^{ \pm}, \theta^{ \pm}\right)$

iii) $\int_{-\varepsilon}^{\varepsilon}\left(e\left(u_{h}\right), \nabla \theta_{h}\right) \mathrm{d} x_{3}$ converges strongly in $L^{2}\left(S ; S^{3} \times \mathbb{R}^{3}\right)$ toward $\left([u] \otimes_{s} e_{3},[\theta] e_{3}\right)$ if $(\bar{\mu}, \bar{\kappa}) \in(0,+\infty]^{2}$,

iv) $\left(u_{h}, \theta_{h}\right)$ converges strongly in $L^{2}\left(\Omega ; \mathbb{R}^{3} \times \mathbb{R}\right)$ toward $(u, \theta)$,

v) $v_{h}$ converges strongly in $L^{2}\left(\Omega ; \mathbb{R}^{3}\right)$ toward $v$.

Lastly we conclude by using the classical Trotter's theory of convergence of linear semi-groups [4], where it suffices to make hypothesis $\left(\mathrm{H}_{4}\right)$ on the initial state and loading and to establish the following convergence result involving stationary problems:

Proposition 4.3. Under the additional assumption

$$
\left(H_{3}\right): \quad \operatorname{supp}\left(g^{M}\right) \cap \bar{B}_{\varepsilon_{0}}=\operatorname{supp}\left(g^{T}\right) \cap \bar{B}_{\varepsilon_{0}}=\emptyset, \quad \forall t \in[0, T]
$$

and if

$$
\begin{aligned}
& \min \left\{\mathcal{H}^{2}\left(\Gamma_{0}^{M^{+}}\right), \mathcal{H}^{2}\left(\Gamma_{0}^{M^{-}}\right)\right\}=0, \quad \operatorname{say} \mathcal{H}^{2}\left(\Gamma_{0}^{M^{-}}\right)=0, \quad \text { then } \operatorname{supp}\left(g^{M}\right) \cap\left(\partial \Omega_{\varepsilon_{0}}^{-}\right)=\emptyset \\
& \min \left\{\mathcal{H}^{2}\left(\Gamma_{0}^{T^{+}}\right), \mathcal{H}^{2}\left(\Gamma_{0}^{T^{-}}\right)\right\}=0, \quad \operatorname{say} \mathcal{H}^{2}\left(\Gamma_{0}^{T^{-}}\right)=0, \quad \text { then } \operatorname{supp}\left(g^{T}\right) \cap\left(\partial \Omega_{\varepsilon_{0}}^{-}\right)=\emptyset
\end{aligned}
$$

we have

$$
\begin{cases}\text { i) } & \lim _{h \rightarrow h^{*}}\left|{ }^{I} P_{h}\left(I-{ }^{I} A\right)^{-1} \phi-\left(I-A_{h}\right)^{-1} P_{h} \phi\right|_{h}=0, \quad \forall \phi \in{ }^{I} H \\ \text { ii) } & \lim _{h \rightarrow h^{*}}\left|{ }^{I} P_{h}{ }^{I} U^{e}(t)-U_{h}^{e}(t)\right|_{h}=0 \quad \text { uniformly on }[0, T] \\ \text { iii) } & \lim _{h \rightarrow h^{*}} \int_{0}^{T}\left|{ }^{I} P_{h}{ }^{I} F(t)-F_{h}(t)\right|_{h} \mathrm{~d} t=0\end{cases}
$$

Proof. Points ii) and iii) are obtained by an obvious variant, taking into account the increments of temperature $\theta_{h}^{e}$, ${ }^{I} \theta$, of the proof given in [2]. By taking advantage of Proposition 3.1 and (23) it remains to establish the convergence of $\bar{z}_{h}=\left(\bar{v}_{h}, \bar{\theta}_{h}\right)$ solution to (13) with $\phi_{h}={ }^{I} P_{h} \phi$ toward ${ }^{I} \bar{z}=\left({ }^{I} \bar{v},{ }^{I} \bar{\theta}\right)$ solution to (23). By choosing $(v, \theta)=\left(\bar{v}_{h}, \bar{\theta}_{h}\right)$ in $(13)$ we deduce that $\sum_{i=1}^{2}\left(\bar{v}_{h}, \bar{v}_{h}\right)_{h, i}+\left(\bar{\theta}_{h}, \bar{\theta}_{h}\right)_{h, i+2}$ remains bounded and consequently that there exists $z^{*}=\left(v^{*}, \theta^{*}\right)$ in ${ }^{I} Z$ and a non relabeled subsequence such that

$$
\left\{\begin{array}{l}
\text { i) } \bar{z}_{h} \text { converges strongly in } L^{2}\left(\Omega ; \mathbb{R}^{3} \times \mathbb{R}\right) \text { toward } z^{*} \\
\text { ii) } \mathbb{1}_{\Omega_{\varepsilon}}\left(e\left(\bar{v}_{h}\right), \nabla \bar{\theta}_{h}\right) \text { weakly converges in } L^{2}\left(\Omega \backslash S ; S^{3} \times \mathbb{R}^{3}\right) \text { toward }\left(e\left(v^{*}\right), \nabla \theta^{*}\right) \\
\text { iii) } \int_{-\varepsilon}^{\varepsilon}\left(e\left(\bar{v}_{h}\right), \nabla \bar{\theta}_{h}\right) \mathrm{d} x_{3} \text { converges strongly in } L^{2}\left(S ; S^{3} \times \mathbb{R}^{3}\right) \text { toward }\left(\left[v^{*}\right] \otimes_{s} e_{3},\left[\theta^{*}\right] e_{3}\right) \\
\text { iv) the traces on } S_{\varepsilon}^{ \pm} \text {of } \bar{z}_{h} \text {, identified with elements of } L^{2}\left(S ; \mathbb{R}^{3} \times \mathbb{R}\right), \text { converge strongly in } \\
\\
L^{2}\left(S ; S^{3} \times \mathbb{R}^{3}\right) \text { to the traces of } z^{* \pm}
\end{array}\right.
$$

Thus it is easy, taking into account Proposition 4.1-iii) to go to the limit on the various terms of $\Psi_{h}\left(\bar{z}_{h},\left({ }^{I_{1}} P_{h} v, I_{2} P_{h} \theta\right)\right)$ for all $(v, \theta)$ in ${ }^{I} Z$. The novelty, induced by the thermoelastic coupling, lies in terms like $\gamma \int_{B_{\varepsilon}} \operatorname{tr} e\left(\bar{v}_{h}\right)^{I_{2}} P_{h} \theta \mathrm{d} x$ and $\gamma \int_{B_{\varepsilon}} \bar{\theta}_{h} \operatorname{tr} e\left({ }^{I_{1}} P_{h} v\right) \mathrm{d} x$. The convergence of the first term toward $\gamma^{*} \int_{S}\left[v^{*}\right]_{3} \frac{\left(\theta^{+}+\theta^{-}\right)}{2} \mathrm{~d} \hat{x}$ stems from Proposition 4.1-iii) and 
(35)-iii) while the convergence of the second one toward $\gamma^{*} \int_{S} \frac{\left(\theta^{*+}+\theta^{*-}\right)}{2}[v]_{3} \mathrm{~d} \hat{x}$ stems from an integration by part, Proposition 4.1-iii) and (35)-iii), iv). Hence one deduces that $z^{*}={ }^{I} \bar{z}$, so that the whole sequence $\bar{z}_{h}$ converges toward ${ }^{I} \bar{z}$. Then it is straightforward to complete the proof by using the definition of ${ }^{I} P_{h}$, Proposition 4.1 and (35).

Thus we deduce the convergence, uniformly on $[0, T]$, in the sense of Trotter of the solution to (14) toward that to (25) with ${ }^{I} U^{r, 0}={ }^{I} U^{0}-{ }^{I} U^{e}(0)$ and the additional condition of convergence and compatibility between the initial state and loading:

$$
\left(H_{4}\right): \quad{ }^{I} U^{0} \in{ }^{I} U^{e}(0)+D\left({ }^{I} A\right) ; \quad U_{h}^{0} \in U_{h}^{e}+D\left(A_{h}\right) \quad \text { and } \quad \lim _{h \rightarrow h^{*}}\left|{ }^{I} P_{h}{ }^{I} U^{0}-U_{h}^{0}\right|_{h}=0
$$

This can be rephrased in a more explicit way with respect to $\left(\mathcal{P}_{h}\right)$ as

Theorem 4.1. The solution to

$$
\frac{\mathrm{d} U_{h}}{\mathrm{~d} t}+A_{h}\left(U_{h}-U_{h}^{e}\right)=\left(u_{h}^{e}, 0, f_{h}\right), \quad U_{h}(0)=U_{h}^{0}
$$

converges toward

$$
\frac{\mathrm{d}^{I} U}{\mathrm{~d} t}+{ }^{I} A\left({ }^{I} U-{ }^{I} U^{e}\right)=\left({ }^{I} u^{e}, 0, \frac{f}{\rho}\right), \quad{ }^{I} U(0)={ }^{I} U^{0}
$$

in the sense

$$
\lim _{h \rightarrow h^{*}}\left|{ }^{I} P_{h} U(t)-{ }^{I} U(t)\right|_{h}=0, \quad \lim _{h \rightarrow h^{*}}\left|U_{h}(t)\right|_{h}={ }^{I}\left|{ }^{I} U(t)\right|, \quad \text { uniformly on }[0, T]
$$

\section{Concluding remarks}

Theorem 4.1 implies that ${ }^{I} z=\left({ }^{I} u,{ }^{I} \theta\right)$ satisfies

$$
\begin{aligned}
& \left(\frac{\mathrm{d}^{2 I} u}{\mathrm{~d} t^{2}}, u\right)_{2}+{ }^{I}\left({ }^{I} u, u\right)_{1}-{ }^{I}\left(u,{ }^{I} \theta\right)_{5}=\int_{\Omega} f \cdot u \mathrm{~d} x+\int_{\Gamma_{1}^{M}} g^{M} \cdot u \mathrm{~d} \mathcal{H}_{2}, \quad \forall u \in{ }^{I} V \\
& \left(\frac{\mathrm{d}^{I} \theta}{\mathrm{d} t}, \theta\right)_{4}+{ }^{I}\left({ }^{I} \theta, \theta\right)_{3}+\left(\frac{\mathrm{d}^{I} u}{\mathrm{~d} t}, \theta\right)_{5}=\int_{\Gamma_{1}^{T}} g^{T} \cdot \theta \mathrm{d} \mathcal{H}_{2}, \quad \forall \theta \in{ }^{I} W
\end{aligned}
$$

Thus the limit behavior deals with the transient response to the initial loading $\left(f, g^{M}, g^{T}\right)$ of the assembly of two linearly thermoelastic bodies occupying $\Omega^{ \pm}$as reference configuration and linked along $S$ by a thermomechanical constraint that strongly depends on the relative magnitude of the parameters $(\varepsilon, \lambda, \mu, \kappa, \gamma)$. If we denote by ${ }^{I} \sigma$ and ${ }^{I} q$ the stress vector on $S$ and the heat flux across $S$ and by $w_{T}$ and $w_{N}$ the tangential and normal components of any vector $w\left(w_{N}=w \cdot e_{3}\right.$, $\left.w_{T}=w-w_{N} e_{3}\right)$, the constitutive equations of the constraint read as:

$$
\begin{aligned}
& I=(1,1): \quad{ }^{I} \sigma_{T}=\bar{\mu}\left[{ }^{I} u\right]_{T}, \quad{ }^{I} \sigma_{N}=(\bar{\lambda}+2 \bar{\mu})\left[{ }^{I} u\right]_{N}-\gamma^{*} \frac{\left({ }^{I} \theta^{+}+{ }^{I} \theta^{-}\right)}{2}, \quad{ }^{I} q_{N}=\bar{\kappa}\left[{ }^{I} \theta\right]+\frac{\gamma^{*}}{2}\left[\frac{\mathrm{d}^{I} u}{\mathrm{~d} t}\right]_{N} \\
& I=(2,1): \quad\left[{ }^{I} u\right]_{N}=0, \quad{ }^{I} \sigma_{T}=\bar{\mu}\left[{ }^{I} u\right]_{T}, \quad{ }^{I} q_{N}=\bar{\kappa}\left[{ }^{I} \theta\right] \\
& I=(3,1): \quad\left[{ }^{I} u\right]=0, \quad{ }^{I} q_{N}=\bar{\kappa}\left[{ }^{I} \theta\right] \\
& I=(1,2): \quad{ }^{I} \sigma_{T}=\bar{\mu}\left[{ }^{I} u\right]_{T}, \quad{ }^{I} \sigma_{N}=(\bar{\lambda}+2 \bar{\mu})\left[{ }^{I} u\right]_{N}-\gamma^{* I} \theta, \quad\left[{ }^{I} \theta\right]=0, \quad{ }^{I} q_{N}=\frac{\gamma^{*}}{2}\left[\frac{\mathrm{d}^{I} u}{\mathrm{~d} t}\right]_{N} \\
& I=(2,2): \quad\left[{ }^{I} u\right]_{N}=0, \quad{ }^{I} \sigma_{T}=\bar{\mu}\left[{ }^{I} u\right]_{T}, \quad\left[{ }^{I} \theta\right]=0, \quad{ }^{I} q_{N}=0 \\
& I=(3,2): \quad\left[{ }^{I} u\right]=0, \quad\left[{ }^{I} \theta\right]=0, \quad{ }^{I} q_{N}=0
\end{aligned}
$$

When $I=(1,1)$, a term of thermal nature involving the average temperature has to be added to the classical expression of the normal stress in pure elasticity, while it appears a thermal surface source term proportional to the jumps of temperature and also of normal velocity. When $I=(1,2)$, there are no jump of temperature, the additional term in the normal stress is then proportional to the surface temperature and it appears a thermal surface source proportional to the jump of normal velocity. Note that in these sole cases where thermoelastic coupling is still present in the constraint, Lamé coefficients $\lambda$ and $\mu$ should be of order $\varepsilon$ and thus $\tilde{\alpha}$ of order $1 / \varepsilon$ in a way that $\gamma^{*}=\lim (3 \lambda+2 \mu) \tilde{\alpha}$ is finite and positive.

Lastly, as usual, our proposal of a simplified but accurate enough model for the behavior of the real structure is the one obtained in the case $I=(1,1)$ by replacing $\bar{\lambda}, \bar{\mu}, \bar{\kappa}$ and $\gamma^{*}$ by the real values $\lambda / 2 \varepsilon, \mu / 2 \varepsilon, \tilde{\kappa} / 2 \varepsilon$ and $(3 \lambda+2 \mu) \tilde{\alpha}$. 


\section{References}

[1] C. Licht, A. Léger, F. Lebon, Dynamics of elastic bodies connected by a thin adhesive layer, in: Ultrasonic Wave Propagation in Non Homogeneous Media,

A. Léger, M. Deschamps (Eds.), Springer Proc. Phys., vol. 128, Springer, Heidelberg, Germany, 2009.

[2] C. Licht, A. Léger, S. Orankitjaroen, A. Ould Khaoua, Dynamics of elastic bodies connected by a thin soft viscoelastic layer, J. Math. Pures Appl. 99 (6)(2013) 685-703.

[3] H. Brézis, Functional Analysis, Sobolev Spaces and Partial Differential Equations, Springer, 2011.

[4] H.F. Trotter, Approximation of semi-groups of operators, Pacific J. Math. 28 (1958) 897-919.

[5] C. Licht, S. Orankitjaroen, Dynamics of elastic bodies connected by a thin soft inelastic layer, C. R. Mecanique 341 (2013) $323-332$. 